\title{
Enhancement of the critical current density in Chevrel phase superconducting wires
}

\author{
N. Cheggour, M. Decroux, A. Gupta, and Ø. Fischer \\ DPMC, Université de Genève, 1211 Genève 4, Switzerland
}

\section{J. A. A. J. Perenboom}

High Field Magnet Laboratory and Research Institute for Materials, University of Nijmegen, Toernooiveld, NL-6525 ED Nijmegen, The Netherlands

\author{
V. Bouquet, M. Sergent, and R. Chevrel \\ Laboratoire de Chimie du Solide et Inorganique Moléculaire, Université de Rennes, France
}

(Received 11 November 1996; accepted for publication 14 January 1997)

\begin{abstract}
The critical current densities $J_{c}$ of Chevrel phase wires with niobium as an antidiffusion barrier were measured in magnetic fields up to $24 \mathrm{~T}$. At $20 \mathrm{~T}$ and $1.9 \mathrm{~K}, J_{c}$ reaches $5.4 \times 10^{8} \mathrm{~A} / \mathrm{m}^{2}$ and decreases slightly down to $3.1 \times 10^{8} \mathrm{~A} / \mathrm{m}^{2}$ at $24 \mathrm{~T}$. A wire with a $20 \%$ superconducting cross section has been successfully drawn and its overall critical current density $J_{c}^{\mathrm{ov}}$ exceeds $100 \mathrm{~A} / \mathrm{mm}^{2}$ at $1.9 \mathrm{~K}$ up to a magnetic field slightly above $20 \mathrm{~T}$. This demonstrates the ability of Chevrel phase wires to be used in high magnetic field applications. Moreover, some parts of the coil have certainly higher $J_{c}$, since $J_{c}$ is very often limited by a thermal excursion of the entire coil. The effective upper critical field $\mu_{0} H_{c 2}^{*}$, deduced from the magnetic field dependence of $J_{c}$, is too low compared to the expected bulk value, indicating that superconducting properties at the grain boundaries are still degraded. If the bulk $\mu_{0} H_{c 2}$ can be restored at the grain surfaces, $J_{c}^{\mathrm{ov}}$ should be higher than $100 \mathrm{~A} / \mathrm{mm}^{2}$ up to at least 30 T. (C) 1997 American Institute of Physics. [S0021-8979(97)00409-X]
\end{abstract}

\section{INTRODUCTION}

The development of superconducting cables to produce ultrahigh magnetic fields is an actual point of interest of specialized industries. Hybrid superconducting magnets using $\mathrm{NbTi}$ and $\mathrm{Nb}_{3} \mathrm{Sn}$ materials allow to obtain steady-state magnetic fields slightly above $22 \mathrm{~T}$. However, it is established that with these materials, the generation of higher magnetic fields can hardly be achieved, since $\mathrm{Nb}_{3} \mathrm{Sn}$ is getting close to its intrinsic upper critical field. Therefore, the development of a suitable technology with superconducting materials having higher upper critical field is essential. With a critical field of $55 \mathrm{~T}$ at $4.2 \mathrm{~K}$, the $\mathrm{PbMo}_{6} \mathrm{~S}_{8}$ Chevrel phase (CP) material largely satisfies this requirement. ${ }^{1}$ Recently, we have reported that the critical current densities $J_{c}$ at $15 \mathrm{~T}$, reached $5.0 \times 10^{8} \mathrm{~A} / \mathrm{m}^{2}$ and $8.1 \times 10^{8} \mathrm{~A} / \mathrm{m}^{2}$ at 4.2 and $1.9 \mathrm{~K}$, respectively. ${ }^{2}$ These results were obtained on wires having a small superconducting cross section $(6 \%-10 \%)$. To demonstrate that Chevrel phase wires are still candidates for the third generation of superconducting cables, it is important to realize a wire with an overall critical current density $J_{c}^{o v}$ exceeding $100 \mathrm{~A} / \mathrm{mm}^{2}$ up to magnetic field higher than $20 \mathrm{~T}$.

To reach this goal, the powder synthesis and the wire manufacturing have to be kept under full control in order to achieve the reproducibility needed to highlight the basic problems limiting the $J_{c}$ performance. Generally, the powder synthesis is done by a solid state reaction in sealed quartz tubes which leads to a contamination of CP grains by oxygen. Our effort was concentrated on the development of a powder synthesis method in a sealed molybdenum crucible, starting from the $\mathrm{Mo}_{6} \mathrm{~S}_{8}$ precursor. This new process ${ }^{3,4}$ has strongly improved the quality of the powder and the reproducibility of the grain morphology which is an essential parameter during the deformation process. ${ }^{5}$
The Chevrel phase wires are made by using the powderin-tube method which consists of a CP powder core contained in an antidiffusion barrier ( $\mathrm{Nb}, \mathrm{Mo}$, or $\mathrm{Ta}$ ) surrounded by an outer jacket made of copper or stainless steel (SS) ${ }^{6-8}$ With niobium, a tearing of this sheet ${ }^{5}$ occurs during the deformation of the wire. These defects are the main source of $J_{c}$ fluctuations along the length of the wire. To avoid them, we have looked for harder materials for the outer sheet able to sustain the deformation strain. Among the materials investigated, $\mathrm{CuNi} 30 \%$ is able to considerably reduce the occurrence of holes in the niobium sheet. ${ }^{2}$

The final density of the CP powder in the wire crucially influences the critical current performances. We have observed that the CP density at the end of the drawing has no clear correlation with its initial value. ${ }^{5}$ Because of the nonductility of $\mathrm{CP}$ grains at room temperature, a too large powder densification prior to the drawing has to be avoided in order to preserve a certain "fluidity" needed to prevent local overdeformations of the niobium sheet. This means that a full densification of the powder has to be done after the wire deformation is completed. The heat treatment under hot isostatic pressing (HIP) of the CP wire is a very efficient process to densify the powder core of the wire in its final form. ${ }^{2,6}$ With this technique, a very dense superconducting core is obtained, resulting in an appreciable increase of the critical currents compared those obtained with heat treatments under the same conditions but at ambient pressure. This improvement of $J_{c}$ comes from a better CP powder densification which favors a good sintering but without significant changes, neither on the grain boundary limitations $\left(T_{c}, \mu_{0} H_{c 2}^{*}\right)$, nor on the magnetic flux lines pinning mechanisms. ${ }^{5}$ 
In this work, we present the $J_{c}$ measurements up to magnetic fields of $24 \mathrm{~T}$ on wires similar to those previously investigated. ${ }^{2,45}$ For one of the wires reported here, the superconducting cross section has been increased from $6 \%$ to $10 \%$ to $20 \%$ without altering $J_{c}$, allowing to get record $J_{c}^{o v}$ for Chevrel phase wires.

\section{SAMPLE PREPARATION}

We present hereafter the study of two wires having different chemical compositions for the superconducting powder: $\mathrm{Pb}_{0.2} \mathrm{Sn}_{0.8} \mathrm{Mo}_{6} \mathrm{~S}_{8}+0.2$ at. \% $\mathrm{Sn}$ for wire No. 1 and $\mathrm{Pb}_{0.6} \mathrm{Sn}_{0.4} \mathrm{Mo}_{6} \mathrm{~S}_{8}+0.2$ at. \% $\mathrm{Sn}+10$ wt \% of precursors $(\mathrm{Pb}$, $\mathrm{MoS}_{2}$, Mo) for wire No. 2. These powders were synthesized from the $\mathrm{Mo}_{6} \mathrm{~S}_{8}$ precursor in a sealed molybdenum crucible. $^{2,3}$ The billet consists of a CP core of $10 \mathrm{~mm}$ in a melt processed niobium tube of $14 \mathrm{~mm}$ in external diameter, surrounded by an outer jacket made of $\mathrm{CuNi30 \%}$ with a diameter of $20 \mathrm{~mm}$. The filling of billets with the powder is carried out under secondary vacuum $\left(2 \times 10^{-5}\right.$ Torr $)$ in a special chamber made to first outgas the powder at elevated temperature and then precompress it into the $\mathrm{Nb}$ tube. Once the billet is sealed, the deformation starts with the swaging of the billet to reduce its diameter from $20 \mathrm{~mm}$ down to about 8 $\mathrm{mm}$. Then, we draw the wires using dies having a reduction ratio between $5 \%$ and $15 \%$. At a diameter of $3 \mathrm{~mm}$, wire No. 1 is inserted into a stainless steel tube of $4 \times 3 \mathrm{~mm}$ and drawn down to a diameter of $0.52 \mathrm{~mm}$. Wire No. 2 is drawn down to $2 \mathrm{~mm}$ and the $\mathrm{CuNi} 30 \%$ sheet is chemically removed in diluted nitric acid. The wire is then reinforced with a stainless steel tube of $2 \times 1.5 \mathrm{~mm}$ and finally drawn down to 0.4 $\mathrm{mm}$. With these final diameters, the two wires have the same superconducting cross section. Therefore, the total deformation of the $\mathrm{CP} / \mathrm{Nb}$ composite is the same for both wires and is also identical to all the wires studied previously. ${ }^{2,4,5}$ Longitudinal and transverse micrographs of these wires after the HIP treatment, are presented in Figs. 1 and 2. The excellent regularity of the different materials forming the wire shows the good quality of the deformation achieved with our process. The superconducting core represents $10 \%$ of the total wire cross section for wire No. 1 and $20 \%$ for wire No. 2 . After the HIP treatment, the outer diameter of the wires is always shrunk as a consequence of the powder densification. The diameter of wire No. 1 is reduced from 0.52 to $0.50 \mathrm{~mm}$ and the diameter of wire No. 2 from 0.4 to $0.38 \mathrm{~mm}$. Assuming that this reduction is solely due to the compaction of the powder, the density of the CP core at the end of the deformation can be estimated once the final density (after the HIP) is known. Figures 1 and 2 show that the powder core looks very dense after the HIP process and we can assume that it is close to be fully densified $(100 \%)$. In consequence our estimate for the density at the end of the deformation represents an upper limit. For the wire reinforced with stainless steel, the upper limit of the $\mathrm{CP}$ core density at the end of the deformation is around $75 \%-80 \%$. This corresponds to a very weak increase of the density during the deformation processes since the initial density after the filling of the billet is around $70 \%$.
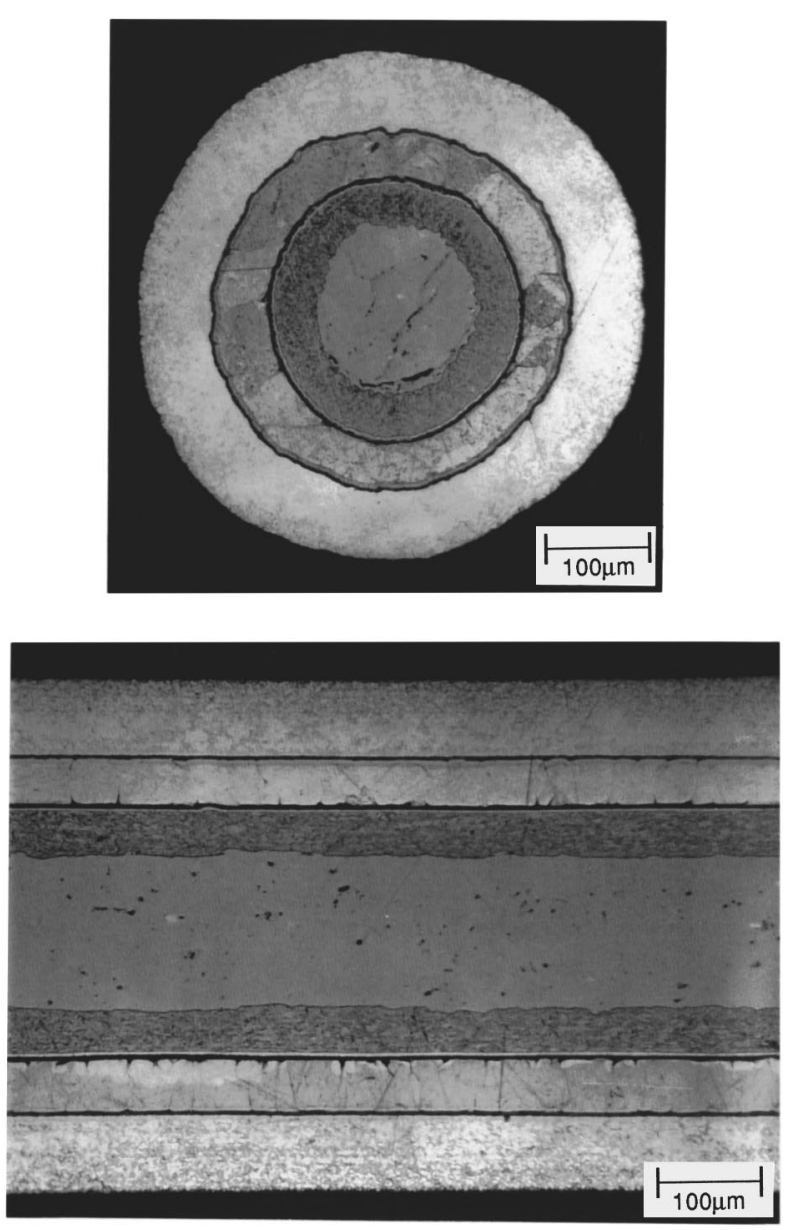

FIG. 1. Transverse and longitudinal micrographs of wire No. $1(\phi=0.50$ $\mathrm{mm}$ ) after the HIP treatment.

The critical current densities of these wires are measured on small coils of 14 turns and are determined with the conventional $1 \mu \mathrm{V} / \mathrm{cm}$ criteria. These coils are first wound on a stainless steel sample holder of $35 \mathrm{~mm}$ diameter and HIP treated for 30 min under $1.9 \mathrm{kbar}$ of argon and $900{ }^{\circ} \mathrm{C}$. Then the coils are carefully transferred to an insulating holder having the same diameter and seven voltage contacts are fixed on every second turn to measure $J_{c}$ and to check its homogeneity over a length of $\sim 1.6 \mathrm{~m}$.

\section{RESULTS}

The critical current results of two representative coils of each wire (one coil with a low $J_{c}$ and one coil with a large $\left.J_{c}\right)$ are presented in Fig. 3 and important values are summarized in Table I. To locate the defects limiting the low $J_{c}$ coils, we have electroplated these coils with copper. These coppered coils will be referred to as wire No. 1, coil No. 2 (coppered) and wire No. 2, coil No. 2 (coppered). The best results were obtained with wire No. 2 which has a chemical composition richer in lead and for which the $\mathrm{CuNi} 30 \%$ sheet has been removed. At $20 \mathrm{~T}, J_{c}$ reaches $2.4 \times 10^{8} \mathrm{~A} / \mathrm{m}^{2}$ and $5.4 \times 10^{8} \mathrm{~A} / \mathrm{m}^{2}$ at 4.2 and $1.9 \mathrm{~K}$, respectively, and at $24 \mathrm{~T}$, $J_{c}$ is $0.8 \times 10^{8} \mathrm{~A} / \mathrm{m}^{2}$ and $3.1 \times 10^{8} \mathrm{~A} / \mathrm{m}^{2}$ at the same temperatures. With this wire, $J_{c}$ at $15 \mathrm{~T}$ was even improved compared to those previously reported ${ }^{2}$ in spite of the rela- 

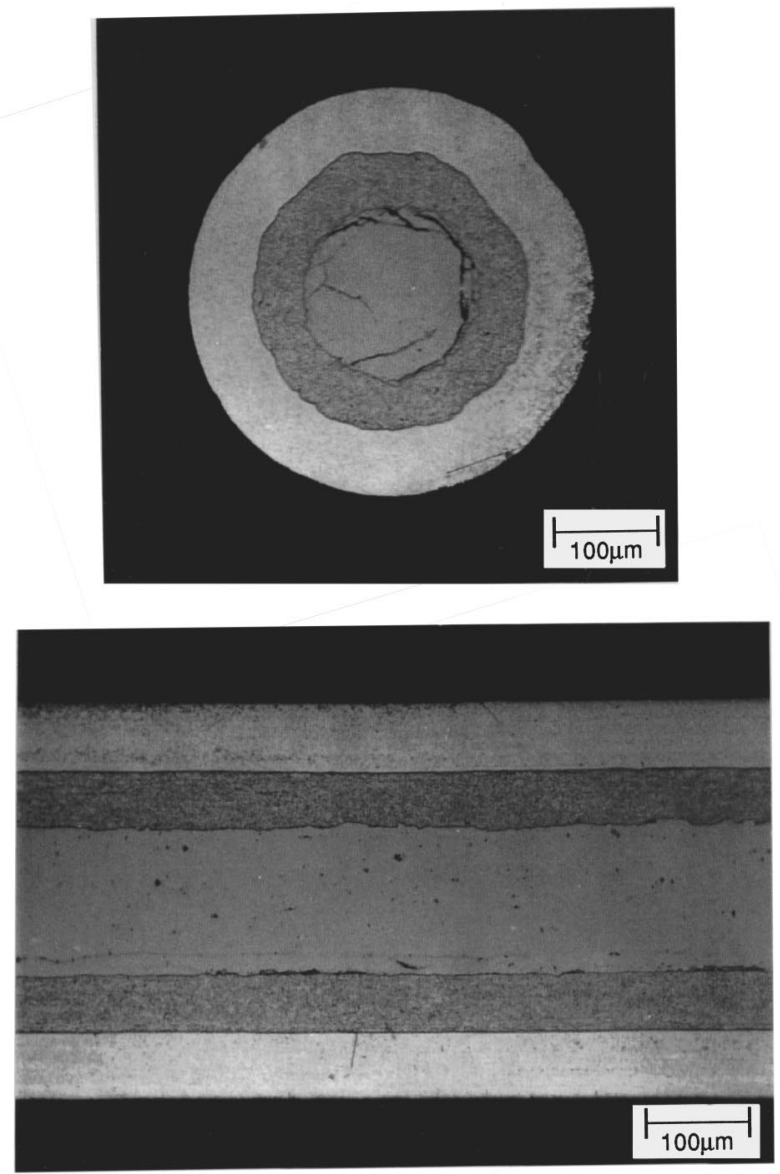

FIG. 2. Transverse and longitudinal micrographs of wire No. $2(\phi=0.38$ $\mathrm{mm}$ ) after the HIP treatment.

tive superconducting cross section having been increased to $20 \%$. The major achievement obtained with this wire is its overall critical current $J_{c}^{\mathrm{ov}}$, which reaches 105 and $63 \mathrm{~A} / \mathrm{mm}^{2}$ at 20 and $24 \mathrm{~T}$, respectively and $1.9 \mathrm{~K}$. For the first time the technological requirement of $100 \mathrm{~A} / \mathrm{mm}^{2}$ is achieved above $20 \mathrm{~T}$ with a Chevrel wire. This improvement of the overall critical current density represents an increase by more than a factor 2 with respect to any results published up to now. ${ }^{6-9}$

\section{DISCUSSIONS}

The improvement in the $J_{c}$ performances of these $\mathrm{CP}$ wires is mainly the consequence of a better understanding of the constraints that the materials forming the wire impose either during the deformation or in the final heat treatment.

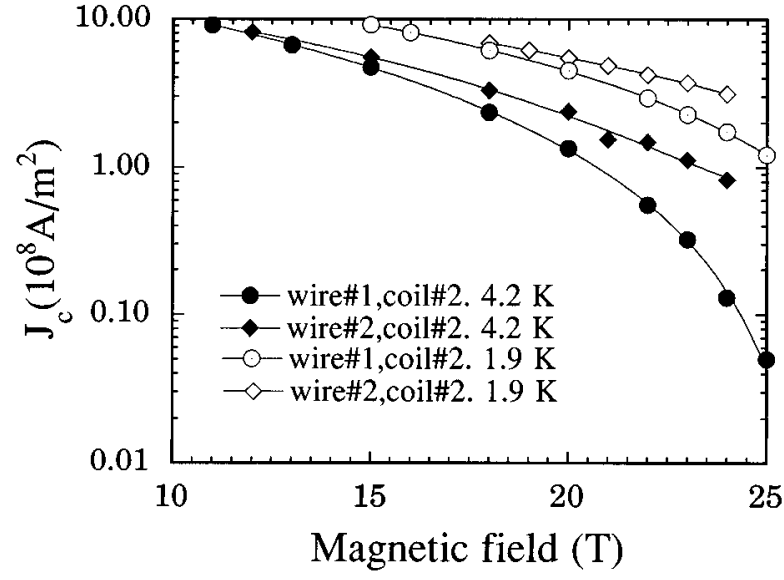

FIG. 3. Magnetic field dependence of $J_{c}$ of wire No. 1 and wire No. 2 up to 24 at 4.2 and $1.9 \mathrm{~K}$.

Among them, the chemical and mechanical compatibility of the antidiffusion barrier with the Chevrel phase were the most important problems we have addressed and our choice of a niobium barrier appears to us as the best compromise.

\section{A. Antidiffusion barrier}

Among the materials used for the antidiffusion barrier in the $\mathrm{CP}$ wires, molybdenum, tantalum, and niobium are frequently used. Molybdenum has the advantage to be chemically inert with respect to the Chevrel phases. ${ }^{6,7}$ However, because of its nonductility at room temperature, the deformation of this material needs to be performed at high temperatures. This produces a sintering of the $\mathrm{CP}$ powder at the beginning of the deformation, making it less "fluid" and leading to fractures either in the barrier or in the superconducting core. With niobium or tantalum, the deformation can be performed at room temperature ${ }^{7-9}$ but these materials are not totally inert with respect to the Chevrel phase or its precursors. A detailed study of the chemical reactivity between $\mathrm{CP}$ precursors $\left(\mathrm{MoS}_{2}, \mathrm{PbS}, \mathrm{Sn}\right)$ and $\mathrm{Nb}$ showed that sulfur, lead, and tin may diffuse toward niobium and form at the interface a layer of $\mathrm{Nb}_{2} \mathrm{~S}_{3}$ intercalated with $\mathrm{Pb}$ or an $A-15 \mathrm{Nb}_{3} \mathrm{Sn}$ phase. The composition of this layer depends on the stoichiometry of the CP powder as well as on the temperature and annealing time, and is nonsuperconducting if $\mathrm{Nb}_{3} \mathrm{Sn}$ is not formed. ${ }^{9}$ We have investigated the reactivity of a $\mathrm{Nb}$ powder mixed with a Chevrel phase at temperatures up to $1200{ }^{\circ} \mathrm{C}$. X-ray analysis did not reveal any traces of niobium sulfide which would indicate the decomposition of the Chevrel phase. However, in a similar study carried out with

TABLE I. Summary of the critical current density $J_{c}$ and the overall critical current density $J_{c}^{\text {ov }}$ results, in case of wire No. 1, coil No. 2 and wire No. 2, coil No. 2 (both coppered).

\begin{tabular}{cccccccc}
\hline \hline Samples & $\begin{array}{c}T \\
(\mathrm{~K})\end{array}$ & $\begin{array}{c}J_{c}(15 \mathrm{~T}) \\
\left(\mathrm{A} / \mathrm{mm}^{2}\right)\end{array}$ & $\begin{array}{c}J_{c}^{\mathrm{ov}}(15 \mathrm{~T}) \\
\left(\mathrm{A} / \mathrm{mm}^{2}\right)\end{array}$ & $\begin{array}{r}J_{c}(20 \mathrm{~T}) \\
\left(\mathrm{A} / \mathrm{mm}^{2}\right)\end{array}$ & $\begin{array}{r}J_{c}^{\mathrm{ov}}(20 \mathrm{~T}) \\
\left(\mathrm{A} / \mathrm{mm}^{2}\right)\end{array}$ & $\begin{array}{r}J_{c}(24 \mathrm{~T}) \\
\left(\mathrm{A} / \mathrm{mm}^{2}\right)\end{array}$ & $\begin{array}{c}J_{c}^{\mathrm{ov}}(24 \mathrm{~T}) \\
\left(\mathrm{A} / \mathrm{mm}^{2}\right)\end{array}$ \\
\hline wire No. 1, coil No. 2 & 4.2 & 470.5 & 47.9 & 133.8 & 13.6 & 13.0 & 1.3 \\
wire No. 1, coil No. 2 & 1.9 & 911.5 & 92.8 & 445.9 & 45.4 & 173.6 & 17.7 \\
wire No. 2, coil No. 2 & 4.2 & 548.2 & 106.3 & 236.8 & 45.9 & 82.3 & 16 \\
wire No. 2, coil No. 2 & 1.9 & $>912$ & $>176$ & 542.7 & 105.3 & 310.9 & 60.3 \\
\hline \hline
\end{tabular}



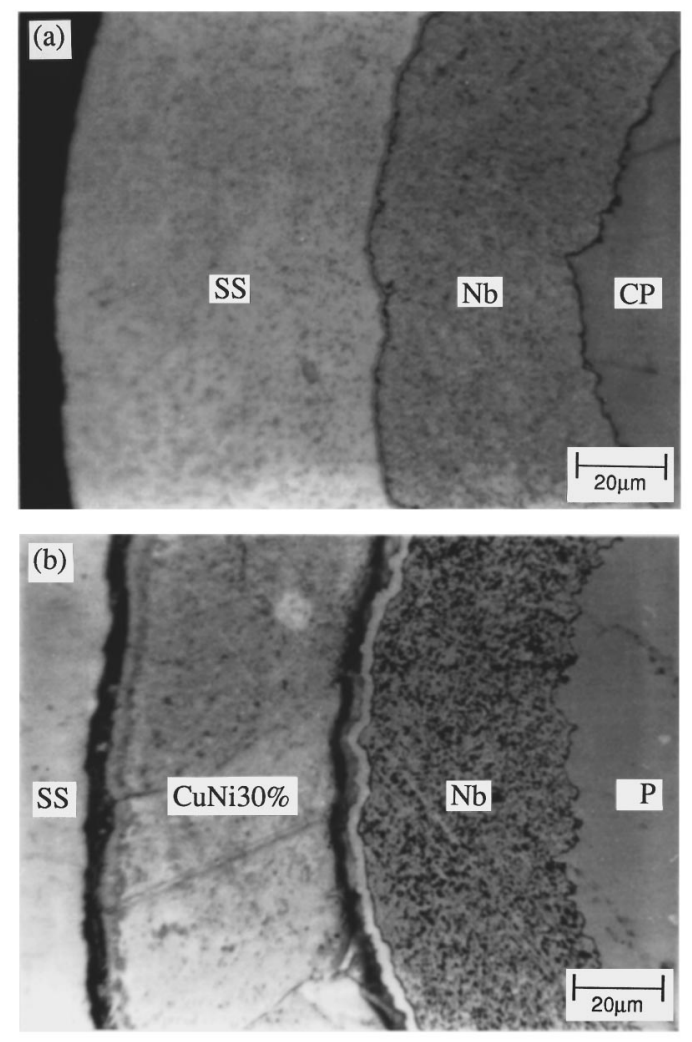

FIG. 4. Enlarged micrograph of wire No. 1 (b) and wire No. 2 (a).

tantalum we observed the formation of $\mathrm{TaS}_{2}$, indicating that the Chevrel phase is unstable against tantalum. This shows that the $\mathrm{Nb}$ barrier represents a good compromise as long as the prereacted powder is pure enough to prevent the formation of the $\mathrm{Nb}-\mathrm{S}-\mathrm{Pb}$ lammellar layer which favors lead losses from the surface of the $\mathrm{CP}$ grains and therefore contributes to the degradation of the superconducting properties at the grain boundaries. ${ }^{3}$ On the other hand it was reported that the reactivity between $\mathrm{Nb}$ and the $\mathrm{CP}$ precursors depends on the temperature, the annealing time and the powder density. ${ }^{9}$ The more dense the powder is, the less important the thickness of the layer between $\mathrm{Nb}$ and the $\mathrm{CP}$ precursors can be. According to this result, we were expecting that HIP heat treatments could markedly reduce the impact of this problem. For the wires studied in the present work, we have performed the HIP heat treatment for $30 \mathrm{~min}$ at $900{ }^{\circ} \mathrm{C}$ and under $1.9 \mathrm{kbar}$ of argon. The good sintering, already achieved at this temperature, is reflected in the high values of
$J_{c}$ obtained for these wires at low magnetic fields. Figure 4 shows an enlarged transverse micrograph of wire No. 1 and wire No. 2. No layer can be observed at the $\mathrm{Nb}-\mathrm{CP}$ interface at this scale indicating that if there is a reaction, it would involve a negligible amount of materials. This confirms the weak reactivity of niobium with pure Chevrel phases. However, it would be desirable to densify the powder prior to the heat treatment in order to decrease further the chemical reactivity between $\mathrm{Nb}$ and the $\mathrm{CP}$. Cold isostatic pressing up to $12 \mathrm{kbar}$ has not lead to an appreciable densification of the superconducting core because of nonductility of the CP powder at room temperature. Therefore, to optimize the temperature-time profile of the HIP treatment, a better knowledge of the mechanical properties of the $\mathrm{CP}$ phase as a function of the temperature is required. Finally, the micrographs of Fig. 4 show the presence of a growing layer at the $\mathrm{NbCuNi} 30 \%$ interface which could be the $\mathrm{Nb}_{3} \mathrm{Ni}$ phase. This indicates that $\mathrm{Ni}$ is diffusing slowly towards the superconducting core and proves the effectiveness of the $\mathrm{Nb}$ sheet which prevents the superconducting core to be contaminated by the Ni magnetic ions. On the other hand, no diffuse layer is observed on the wire with the SS-Nb sheet. Therefore, if the temperature and the annealing time have to be increased to optimize the critical current and its dependence versus magnetic fields, wires with a SS-Nb sheet should be preferred to avoid a Ni contamination.

\section{B. Critical current homogeneity}

The homogeneity of the critical current $I_{c}$ measured at 9 $\mathrm{T}$ and $4.2 \mathrm{~K}$, along the seven sections of six coils investigated in this work, are summarized in Table II. In the case of wire No. 1, coil No. 1 and wire No. 2, coil No. 2, $I_{c}$ looks to be very homogeneous over the whole coil, but it is surprising that the second coil have an $I_{c} 40 \%$ lower. Such a difference between the two coils is too large if one considers that they were made with the same wire and heat treated under the same conditions. The perfect homogeneity showed by the two coils is in fact due to a thermal instability of these wires ${ }^{2}$ which very often show a sudden large voltage jump at $I_{c}$ indicating a thermal runaway. ${ }^{10,11}$ To highlight that these thermal runaways are due to local dissipative defects, we have electroplated with copper, one coil of each wire having a low $I_{c}$. Due to the difficulty to clean perfectly the outer surface of the stainless steel before the electrolysis, the deposition of copper was not perfectly homogenous. The critical current of the coppered coil wire No. 1, coil No. 2, has been

TABLE II. Critical current homogeneity along the seven sections (I-VII) of the six coils measured at $9 \mathrm{~T}$ and $4.2 \mathrm{~K}$. A section corresponds to two turns of the coil.

\begin{tabular}{|c|c|c|c|c|c|c|c|}
\hline Coils & $\begin{array}{c}I_{c}(\mathrm{I}) \\
{[\mathrm{A}]}\end{array}$ & $\begin{array}{c}I_{c}(\mathrm{II}) \\
{[\mathrm{A}]}\end{array}$ & $\begin{array}{c}I_{c}(\mathrm{III}) \\
{[\mathrm{A}]}\end{array}$ & $\begin{array}{c}I_{c}(\mathrm{IV}) \\
{[\mathrm{A}]}\end{array}$ & $\begin{array}{c}I_{c}(\mathrm{~V}) \\
{[\mathrm{A}]}\end{array}$ & $\begin{array}{c}I_{c}(\mathrm{VI}) \\
{[\mathrm{A}]}\end{array}$ & $\begin{array}{c}I_{c}(\mathrm{VII}) \\
{[\mathrm{A}]}\end{array}$ \\
\hline wire No. 1, coil No. 1 & 21.6 & 21.7 & 21.7 & 21.7 & 21.7 & 21.7 & 21.7 \\
\hline wire No. 1, coil No. 2 & 13.2 & 13.2 & 13.2 & 13.2 & 13.1 & 13.1 & 13.1 \\
\hline wire No. 1, coil No. 2, coppered & 22.5 & 13.4 & 13.4 & 23.6 & 23.6 & 23.6 & 23.4 \\
\hline wire No. 2, coil No. 1 & 18.9 & 18.9 & 18.9 & 18.9 & 18.9 & 18.9 & 18.9 \\
\hline wire No. 2, coil No. 2 & 6.6 & 6.6 & 6.6 & 6.7 & 6.7 & 6.7 & 6.7 \\
\hline wire No. 2, coil No. 2, coppered & 21.8 & 6.8 & 23.3 & 23.3 & 23.3 & 23.3 & 23.3 \\
\hline
\end{tabular}


improved in five of the seven sections and reached 23.6 A, a value even better than that of wire No. 1, coil No. 1. However the performance of the best sections of this coil is still limited by the dissipation of defects localized in Secs. II and III. At currents exceeding $13.2 \mathrm{~A}, I-V$ characteristics of these two sections show many small voltage steps of a few millivolts, followed at around $23.6 \mathrm{~A}$ by a large voltage jump indicating the quench of the whole coil. In the other sections of the coil there is a zero-resistance state up to $22.5 \mathrm{~A}$ where a small rounded transition developed in the first section up to the quench current. In case of wire No. 2, we have a similar situation. Both coils have a good homogeneity but with a difference in $I_{c}$ of a factor 2.8. After coppering wire No. 2, coil No. 2, $I_{c}$ is improved by a factor 3.5, with dissipative defects localized in Sec. III. This shows that the critical current values measured in our wires are still limited by extrinsic factors related to electrical and thermal conductivity of the sheet materials of the wires. When the current has to flow through a section of the wire containing a defect, the local current density increases and when it is higher than $J_{c}$, the whole section containing the defect starts to dissipate. If the surrounding materials have good thermal and electrical properties, the heat generated by the dissipative zone can easily be evacuated into the helium bath and the excess current can flow through the sheet materials and in consequence, the dissipative zone will not expand too much. Our wires have external sheets with relatively bad thermal and electrical properties and the heat locally generated can hardly be transferred into the helium bath and the current could not efficiently short circuit this zone. The thin copper layer deposited on the surface of the wires does not improve the thermal environment of the superconducting core, but it provides a better electrical path to short circuit the dissipative zone. In spite of the fact that the current has to cross several interfaces from the superconducting core to the external copper sheet, the situation has been largely improved.

As mentioned in the Introduction, the choice of hard materials for the outer sheets of $\mathrm{CP}$ wires is very important to avoid fractures of the niobium barrier. At the same time, these materials insure to the wire its mechanical stability, necessary to sustain the huge Lorentz force exerted at high magnetic fields. Stainless steel and $\mathrm{CuNi} 30 \%$ are well adapted to fulfill the mechanical constraints but they do not provide good thermal and electrical properties to the wires. CuNi30\% can be used as a sheet material during the drawing and then removed following the procedure we have used for wire No. 2 without altering the $J_{c}$ performances of the wire. At $15 \mathrm{~T}$ this wire has similar $J_{c}$ as a wire drawn from the same billet but for which the reinforcement with stainless steel was made without removing $\mathrm{CuNi} 30 \%$ (wire No. 2 in Ref. 2). To give to CP wires the required mechanical characteristics but also good thermal and electrical properties, CuNi30\% and eventually stainless steel have to be replaced by another hard material with better thermal and electrical conductivity. The preliminary test on the deformation of a wire having a precipitation hardening copper alloy for the outer jacket has been carried out. With $\mathrm{CuNi} 1 \%$ Be $1 \%$ alloys, we have succeeded in the deformation process and the studies of this type of wire are presently under investigations.

\section{Critical current limitations}

One factor responsible for low $J_{c}$ is a poor connectivity of grains due to a weak densification of the superconducting core. By using a HIP heat treatment, we have strongly increased the density of the CP core (Figs. 1 and 2) and therefore the $J_{c}$ at low magnetic field $(<10 \mathrm{~T})$. Nevertheless, the important drop of $J_{c}$ at magnetic fields higher than $20 \mathrm{~T}$ reveals another limitation always encountered in Chevrel phase wires and presently not solved. The magnetic field dependence of $J_{c}$ depends basically on the type of pinning and on the value of the effective upper critical field. In hightemperature superconductors, this effective critical field is identified as the irreversibility field which has its origin in the dynamic properties of a quasi-2D flux line lattice. For Chevrel phases, the possible existence of an irreversibility line has been reported ${ }^{12,13}$ but the proposed $H-T$ phase diagram is not clearly established as an intrinsic property of the flux line lattice of this material. Detailed analysis of the specific heat anomaly of the $\mathrm{PbMo}_{6} \mathrm{~S}_{8}$ Chevrel phase have shown that the superconducting transition temperature in zero magnetic field spreads over several kelvins from $T_{c}$ (14-15 K) down to almost 9-8 K. ${ }^{3,14}$ Since the percentage of degraded superconducting phases was found to be larger for powder with a small grain size, we concluded that the superconducting degradation is located at the grain surface. In the work of Ref. 12, the normalized decay rate of the magnetization measured at a low magnetic field $(0.05 \mathrm{~T})$ is mainly constant from $T_{c}$ down to $9-8 \mathrm{~K}$ and then decreases substantially. This corresponds to the minimal value of $T_{c}$ deduced from the specific heat anomaly. ${ }^{14}$ These degraded regions can be seen as channels ${ }^{15}$ where magnetic flux lines may easily flow and therefore $J_{c}$ will depend primarily on the upper critical field of these channels. Using the simple WHH expression for the upper critical field of dirty superconductors $^{16}$

$$
\mu_{0} H_{c 2}=0.693 T_{c}\left|\frac{d\left(\mu_{0} H_{c 2}\right)}{d T}\right|_{T_{c}}
$$

and assuming a maximal value of $7 \mathrm{~T} / \mathrm{K}$ (Ref. 1) for the initial slope of the upper critical field and a $T_{c}$ for the channels of $8-9 \mathrm{~K}$, we get an upper critical field at zero temperature of 38-43 T. As discussed above, the superconducting properties at the surface of the grains are degraded certainly by a modification of the lead stoichiometry at the surface. ${ }^{3}$ We recall that an intercalation of $\mathrm{Pb}$ into the $\mathrm{Mo}_{6} \mathrm{~S}_{8}$ structure is possible at a temperature as low as $440{ }^{\circ} \mathrm{C},{ }^{17}$ demonstrating the existence of a $\mathrm{Pb}$ mobility into the $\mathrm{Mo}_{6} \mathrm{~S}_{8}$ structure. Since no stoichiometric modification of the $\mathrm{Mo}_{6} \mathrm{~S}_{8}$ structure is expected in this range of temperature, it was concluded that the improved superconducting properties of the intercalated $\mathrm{Pb}-\mathrm{Mo}_{6} \mathrm{~S}_{8}$ phase with time has to be correlated with an increase of the $\mathrm{Pb}$ content into the host structure. ${ }^{18}$ Since the sintering process is carried out at a relatively low temperature and for a short period of time, the resulting dense phase is made of well-connected grains having still degraded superconducting properties at their surface. Once the critical field of these channels is reached, the flux lines should flow along these channels simulating a pseudo-irreversibility field. 


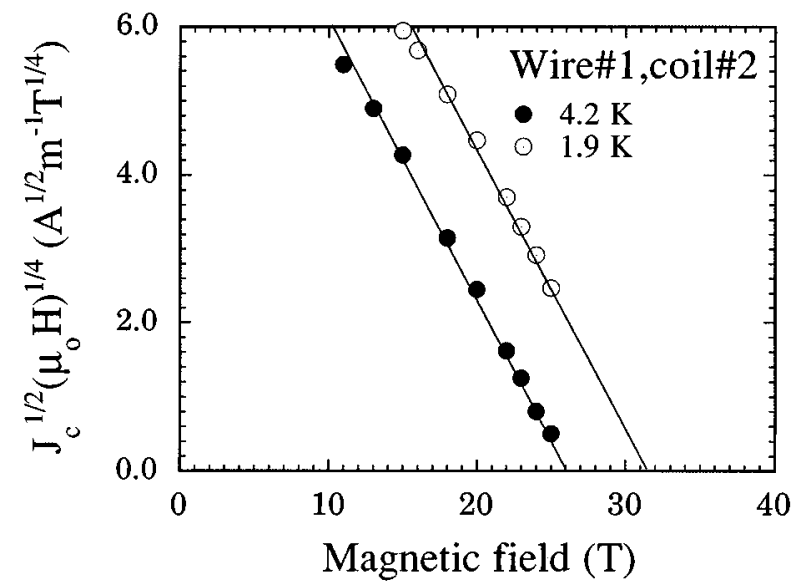

FIG. 5. Kramer plot for wire No. 1, coil No. 2 at 4.2 and $1.9 \mathrm{~K}$.

A detailed investigation of the $I-V$ characteristics of $\mathrm{CP}$ wire with molybdenum barrier confirms the hypothesis that two distinct upper critical fields exist in the superconducting phases. ${ }^{19}$ In order to estimate the effective upper critical field $\mu_{0} H_{c 2}^{*}$ of the wires investigated in this work, Figs. 5 and 6 present $J_{c}^{1 / 2}\left(\mu_{0} H\right)^{1 / 4}$ versus $\mu_{0} H$ (known as the Kramer $\operatorname{plot}^{20}$ ). Wire No. 1 has a $\mu_{0} H_{c 2}^{*}$ of $26 \mathrm{~T}$ at $4.2 \mathrm{~K}$, with an increase up to $31.5 \mathrm{~T}$ at $1.9 \mathrm{~K}$. For wire No. 2 which has a chemical composition richer in lead, we obtain higher values $\left(\mu_{0} H_{c 2}^{*}=31.5\right.$ and $38 \mathrm{~T}$ at 4.2 and $1.9 \mathrm{~K}$, respectively). This difference in $\mu_{0} H_{c 2}^{*}$ between the two wires is reflected in the drop of $J_{c}$ at high magnetic fields (Fig. 3) which is less pronounced in case of wire No. 2. The effective critical field of both wires nevertheless stays far below the bulk values, expected to be at $4.2 \mathrm{~K}$, around 40 and $50 \mathrm{~T}$ for wire No. 1 and wire No. 2, respectively. This means $J_{c}$ could be much higher than values reported here if one could succeed to chemically stabilize the last nanometers of the surface of $\mathrm{CP}$ grains which are degraded.

The question which rises now is how high the $J_{c}$ might be in these wires, if their effective critical field reaches the level of bulk upper critical field. In CP superconductors, the critical current density is generally limited by a shearing of

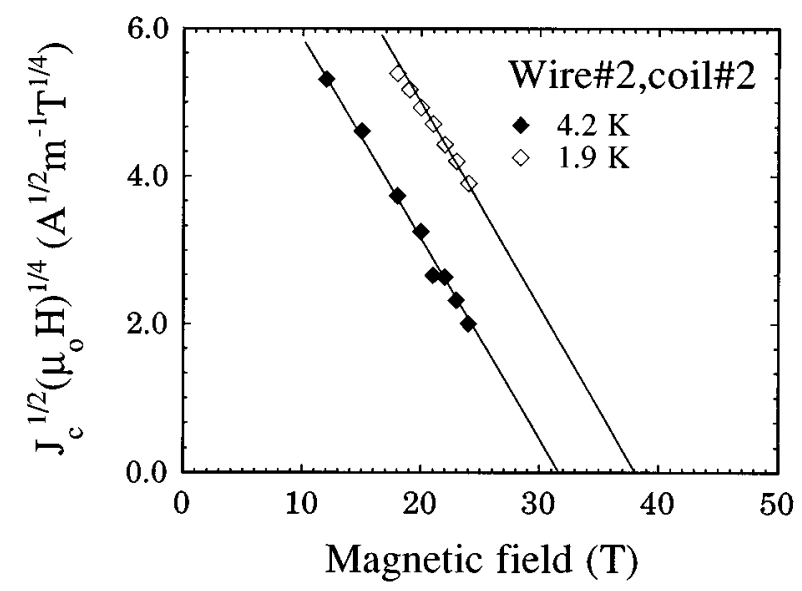

FIG. 6. Kramer plot for wire No. 2, coil No. 2, at 4.2 and $1.9 \mathrm{~K}$.

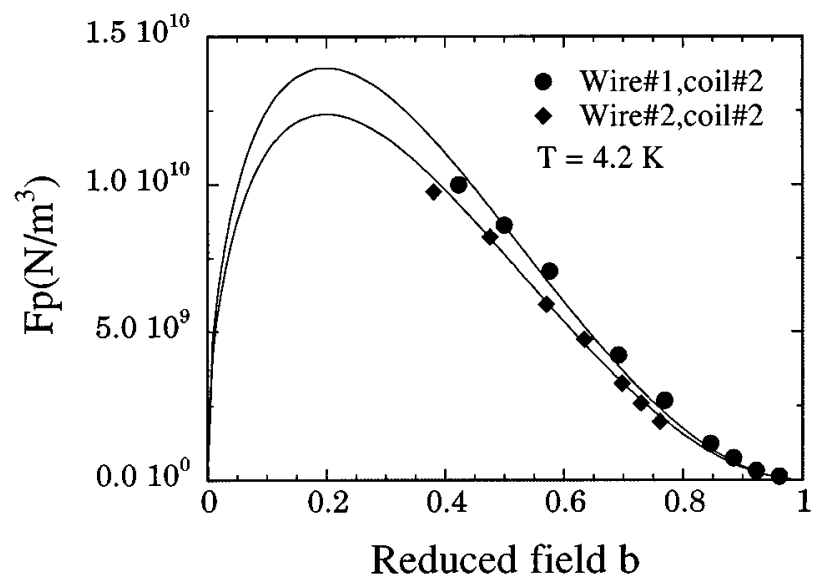

FIG. 7. Reduced magnetic field dependence of the pinning force density $F_{p}$ for wire No. 1, coil No. 2 and wire No. 2, coil No. 2 at 4.2 K. The solid lines represent the prediction of Eq. (2).

the flux line lattice along weak pinning channels, probably located at the degraded grain boundaries. ${ }^{21}$ The volume pinning force $\left(F_{p}\right)$ against the reduced field $b=\mu_{0} H / \mu_{0} H_{c 2}^{*}$ at $4.2 \mathrm{~K}$, where $\mu_{0} H_{c 2}^{*}$ takes values determined previously from the Kramer plots, is shown in Fig. 7. For both wires, $F_{p}$ is well fitted using the scaling function:

$$
F_{p}=J_{c} \mu_{0} H=\alpha\left[\mu_{0} H_{c 2}^{*}(T)\right]^{2.5} b^{0.5}(1-b)^{2} .
$$

This volume pinning force dependence on the reduced magnetic field is generally found in CP superconductors ${ }^{22,23}$ and indicates that the limiting pinning mechanism of flux lines occurs at the grain boundaries. The prefactor $\alpha$ in Eq. (2) $\left(1.4 \times 10^{7}\right.$ for wire No. 1 and $7.8 \times 10^{6}$ for wire No. 2$)$ differs by almost a factor 2 . This parameter is essentially related to the microstructure of the superconductor and can be influenced by the width of flux flow channels, the effective current flow cross section, as well as the mean grain size. ${ }^{21,22}$ Powders used in the processing of both wires were produced with the same technique and have similar grain sizes. HIP treatment of these coils was performed under the same conditions, during the same run and consequently, both wires have similar density. According to that, the difference between wire No. 1 and wire No. 2 could be solely due to the width of flux flow channels which represents the thickness where the superconducting properties are degraded at the grain boundaries. Secondary phases are greatly suppressed at the grain boundaries by the addition of Sn (adjunction or substitution). ${ }^{24-26}$ Indeed, by adding $\mathrm{Sn}$, surface analysis have clearly shown that the ratio content S/Mo can be lowered towards the value of the ideal stoichiometric composition. According to this result, the degraded grain boundaries might be thicker in the case of wire No. 2 compared to wire No. 1 , and could be the origin of the difference in the prefactor seen between the two wires.

Figures 8 and 9 show the magnetic field dependence of $J_{c}$ predicted from Eq. (2) with the effective critical field determined from the plots of the Figs. 6 and 7. $J_{c}$ has been also calculated assuming values of $\mu_{0} H_{c 2}^{*}$ close to those of bulk upper critical field. Assuming $\mu_{0} H_{c 2}^{*}$ of 40 and $50 \mathrm{~T}$ for wire No. 1 and wire No. 2, respectively, $J_{c}$ could be ex- 


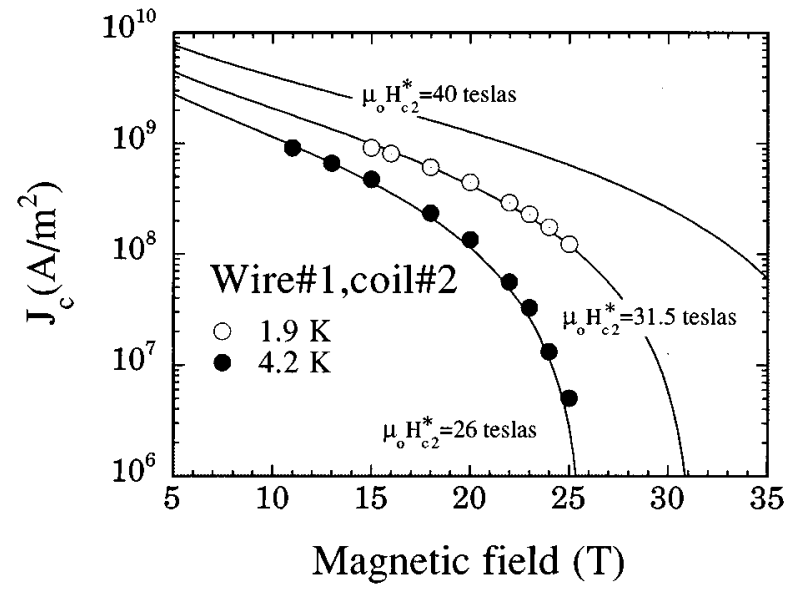

FIG. 8. Calculated $J_{c}\left(\mu_{0} H\right)$ (solid lines) in case of wire No. 1, for different values of the upper critical field.

tremely high. In the case of wire No. $2, J_{c}$ could remain as high as $1 \times 10^{9} \mathrm{~A} / \mathrm{m}^{2}$ up to $25 \mathrm{~T}$. The overall critical current might reach $100 \mathrm{~A} / \mathrm{mm}^{2}$ at a field of $30 \mathrm{~T}$. This indicates that the $\mathrm{CP}$ superconductors may have a great potential which still has to be explored. Finally, we would like to emphasize that $J_{c}$ at $9 \mathrm{~T}$ is around $5 \times 10^{9} \mathrm{~A} / \mathrm{m}^{2}$, a value in good agreement with the estimation of the intragrain critical current density. ${ }^{22,27}$ This agreement gives further evidences that the superconducting properties at the grain boundaries is the main limiting factor in the $J_{c}$ performance of Chevrel phase wires.

\section{CONCLUSION}

In this work, we report very encouraging critical current density results obtained on Chevrel phase superconducting wires. We have successfully drawn a Nb-stainless steel sheeted wire with a superconducting cross section of almost $20 \%$. The critical current density measured on small coils of $\sim 1.6 \mathrm{~m}$ wire length, hot isostatically pressed under $1.9 \mathrm{kbar}$ argon pressure at $900{ }^{\circ} \mathrm{C}$ for $30 \mathrm{~min}$, reached the values of $5.4 \times 10^{8} \mathrm{~A} / \mathrm{m}^{2}$ and $3.1 \times 10^{8} \mathrm{~A} / \mathrm{m}^{2}$ at 20 and $24 \mathrm{~T}$, respec-

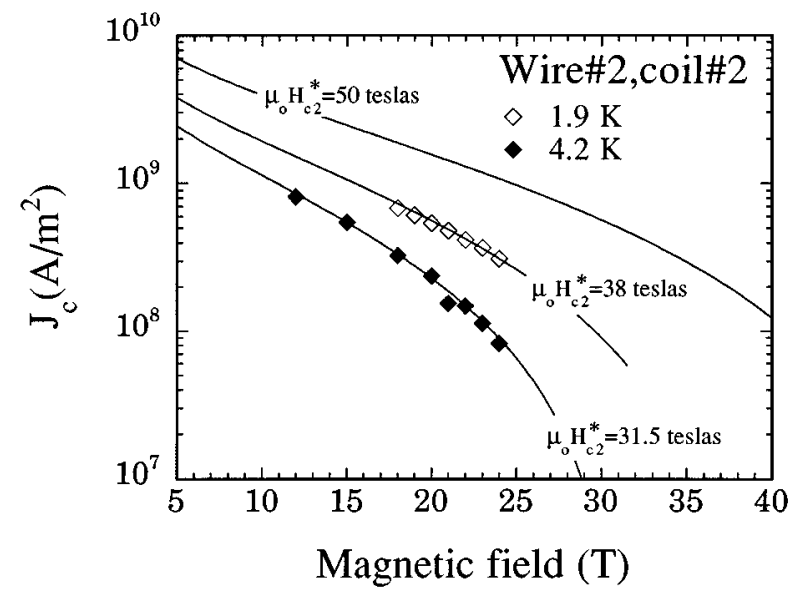

FIG. 9. Calculated $J_{c}\left(\mu_{0} H\right)$ (solid lines) in case of wire No. 2, for different values of the upper critical field. tively, at the temperature of $1.9 \mathrm{~K}$. The overall critical current exceeded 105 and $60 \mathrm{~A} / \mathrm{mm}^{2}$ at the same temperature and fields. These performances are the highest ever reported on this compound at this time and Chevrel phase wires fulfill the essential requirements for high field applications.

With the production of high quality powder which favors a good sintering between the grains at low temperature and short annealing time, and the achievement of high powder densification when using the HIP technique for the wire heat treatment, we have greatly reduced the chemical reactivity with the niobium sheet. Indeed, wire micrographs did not show any evidence of an intermediate layer between $\mathrm{Nb}$ and the CP component. On the other hand, we have observed thermal excursions during $J_{c}$ measurements, which are a manifestation of poor thermal and electrical conductivity of sheet materials. CuNi30\% and stainless steel do not fill all the conditions required for a good thermal stability of the Chevrel phase wires but precipitation hardening alloys as $\mathrm{CuBe}$ may provide an interesting alternative.

The analysis of the magnetic field dependence of $J_{c}$ shows that the effective upper critical field is too low compared to the expected bulk values, and the pinning force is limited by a flux line shear mechanism. This may be understood if we assume that the superconducting properties at grain boundaries are degraded and therefore are the major $J_{c}$ limiting factor. We have seen between investigated wires, a difference of almost a factor of 2 in the prefactor of the pinning force scaling function. This can be attributed to a difference in the thicknesses of the degraded layer at the grain, which is expected to be smaller in the case of the wire No. 1 with a composition richer in Sn. If these channels can have an effective upper critical field close to the bulk value, the calculated $J_{c}$ indicates the great potential of improvement for these CP wires. Chevrel phase wires might be used for high field applications up to at least $30 \mathrm{~T}$, this expectation being based on a wire having a superconducting cross section of $20 \%$. For the purpose of achieving that, the relevant challenge to chemically stabilize the last nanometers of the surface of grains has to be raised by researchers in the future.

\section{ACKNOWLEDGMENTS}

This work is supported by the Commission (Suisse) pour l'Encouragement de la Recherche Scientifique (CERS) within EUREKA. We are grateful to F. Liniger for the wire micrographs and to J. L. Mas for the achievement of the HIP treatment cycle. We also thank H.A.M. Balster, University of Nijmegen, for his assistance during the high field measurements.

\footnotetext{
${ }^{1}$ M. Decroux and $\emptyset$. Fischer, Superconductivity in Ternary Compounds II, Topics in Current Physics (Springer, Berlin, 1982), Vol. 34, pp. 57-98.

${ }^{2}$ N. Cheggour, A. Gupta, M. Decroux, R. Flükiger, Ø. Fischer, H. Massat, P. Langlois, V. Bouquet, R. Chevrel, and M. Sergent, Physica C 258, 21 (1996).

${ }^{3}$ M. Decroux, P. Selvam, J. Cors, B. Seeber, Ø. Fischer, R. Chevrel, M. Sergent, and P. Rabiller, IEEE Trans. Appl. Supercond. 3, 1502 (1993).

${ }^{4}$ N. Cheggour, M. Decroux, A. Gupta, S. Ritter, V. Schröter, B. Seeber, R. Flükiger, Ø. Fischer, S. Boudjada, L. Burel, V. Bouquet, R. Chevrel, M. Sergent, H. Massat, P. Langlois, and P. Genevey, Proc. ICMAS-93, 403 (1993).
} 
${ }^{5}$ N. Cheggour, A. Gupta, M. Decroux, J. A. A. J. Perenboom, P. Langlois, H. Massat, R. Flukiger, and $\varnothing$. Fischer, Inst. Phys. Conf. Ser. 148, 507 (1995).

${ }^{6}$ H. Yamasaki, M. Umeda, and S. Kosaka, J. Appl. Phys. 72, 1 (1992).

${ }^{7}$ B. Seeber, P. Hermann, L. Schellenberg, and J. Zuccone, IEEE Trans. Magn. 27, 1108 (1991).

${ }^{8}$ G. Rimikis, W. Goldacker, W. Specking, and R. Flükiger, IEEE Trans. Magn. 27, 1116 (1991).

${ }^{9}$ P. Rabiller, Ph.D. thesis, University of Rennes, 1991.

${ }^{10}$ P. F. Hermann, Ph.D. thesis, University of Geneva, 1990.

${ }^{11}$ P. F. Hermann, L. Schellenberg, J. Zuccone, B. Seeber, Ø. Fischer, R. Grill, and J. A. A. J. Perenboom, Physica C 202, 61 (1992).

${ }^{12}$ C. Rossel, E. Sandvold, M. Sergent, R. Chevrel, and M. Potel, Physica C 165, 233 (1990)

${ }^{13}$ D. N. Zheng, H. D. Ramsbottom, and D. P. Hampshire, Phys. Rev. B 52, 12931 (1995).

${ }^{14}$ J. Cors, Ph.D. thesis, University of Geneva, 1990.

${ }^{15}$ R. Wördenweber, Cryogenics 32, 1098 (1992).

${ }^{16}$ N. R. Werthamer, E. Helfand, and P. C. Höhenberg, Phys. Rev. 147, 295 (1966).

${ }^{17}$ S. Even-Boudjada, Ph.D. thesis, University of Rennes, 1994.

${ }^{18}$ P. Rabillier, M. Rabillier-Baudry, S. Even-Boudjada, L. Burel, R. Chevrel,
M. Sergent, M. Decroux, J. Cors, and J. L. Maufras, Mater. Res. Bull. 29, 567 (1994).

${ }^{19}$ B. Seeber, N. Cheggour, J. A. A. J. Perenboom, and R. Grill, Physica C 234, 343 (1994).

${ }^{20}$ E. J. Kramer, J. Appl. Phys. 44, 1360 (1973).

${ }^{21}$ A. Gupta, M. Decroux, P. Selvam, D. Cattani, T. C. Willis, and Ø. Fischer, Physica C 234, 219 (1994).

${ }^{22}$ D. Cattani, Ph.D. thesis, University of Geneva, 1990

${ }^{23}$ L. Le Lay, T. C. Willis, and D. C. Larbalestier, Appl. Phys. Lett. 60, 775 (1992).

${ }^{24}$ P. Selvam, D. Cattani, J. Cors, M. Decroux, Ph. Niedermann, S. Ritter, $\varnothing$. Fischer, R. Chevrel, and T. Pech, IEEE Trans. Appl. Supercond. 3, 1575 (1993).

${ }^{25}$ P. Selvam, D. Cattani, J. Cors, M. Decroux, Ph. Niedermann, S. Ritter, $\varnothing$. Fischer, L. Burel, R. Chevrel, P. Rabillier, and M. Sergent, Proc. ICMAS91, 157 (1991).

${ }^{26}$ P. Selvam, D. Cattani, J. Cors, M. Decroux, A. Junod, Ph. Niedermann, S. Ritter, Ø. Fischer, P. Rabiller, and R. Chevrel, J. Appl. Phys. 72, 4232 (1992).

${ }^{27}$ M. Decroux, D. Cattani, J. Cors, S. Ritter, A. Stettler, Ø. Fischer, P. Hermann, L. Schellenberg, J. Zuccone, B. Seeber, P. Rabiller, L. Burel, R. Chevrel, and M. Sergent, Proc. ICMAS'90, 147 (1990). 